Z Epileptol 2013 · 26:172-172

DOI 10.1007/s10309-013-0324-4

Online publiziert: 24. Juli 2013

(c) Springer-Verlag Berlin Heidelberg 2013

\section{U. Bettendorf}

Schwerpunktpraxis Epileptologie, Hirschaid

\section{Laudatio}

\section{Ehrenmitgliedschaft von Professor Dr. Gunter Gross-Selbeck}

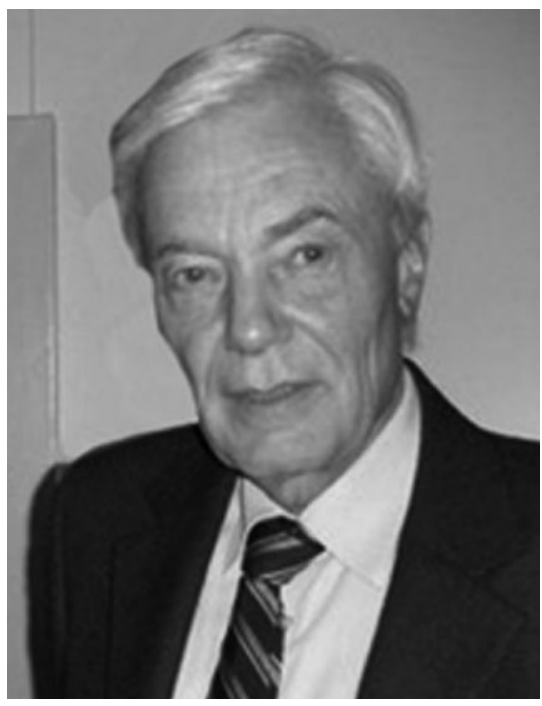

Gunter Gross-Selbeck ist in Kiel aufgewachsen. Nach dem Abitur studierte er ab 1960 in Freiburg, Wien und zuletzt Kiel; hier schloss er 1966 sein Medizinstudium mit der Promotion ab. Seine Medizinalassistentenzeit verbrachte er 1967 und 1968 in Kiel und Bielefeld. Bei Prof. Doose in der Universitätskinderklinik Kiel arbeitete er zunächst als Assistenzarzt und später als Oberarzt. Im Jahr 1978 habilitierte sich Gunter Gross-Selbeck (Thema: „Untersuchungen zur Persönlichkeitsstruktur von Kindern und Jugendlichen mit elektroenzephalographisch nachweisbarer Photosensibilität") und wurde stellvertretender Direktor der Kinderneurologischen Klinik an der Universität Kiel. Ab 1979 war er als Chefarzt des Kinderneurologischen Zentrums der Stadt Düsseldorf am Krankenhaus Gerresheim tätig; hier arbeitete er über 25 Jahre (bis 2004) als Kinder- arzt und begründete die heute etablierte Sozialpädiatrie mit. Die Ernennung zum außerordentlichen Professor an der Universität Düsseldorf in Kooperation mit der Universität Düsseldorf erfolgte 1984.

Gunter Gross-Selbeck wurde 1995 Präsident der Deutsch-ÖsterreichischSchweizerischen Gesellschaft für Neuropädiatrie. Von 2001-2003 war er Präsident der Deutschen Sektion der Internationalen Liga gegen Epilepsie und damals auch Tagungspräsident der Dreiländertagung 2003 in Berlin. Professor Gross-Selbeck ging im Mai 2004 in den Ruhestand und wurde Ehrenmitglied der DeutschÖsterreichisch-Schweizerischen Gesellschaft für Neuropädiatrie.

Ruhestand bedeutete nicht, „sich zur Ruhe setzen“! Seit 2004 bis heute war Gunter Gross-Selbeck als Unterstützer und Mitarbeiter in einer Rehabilitationseinrichtung für Kinder und Jugendliche in Nepal aktiv (bisher insgesamt ca. 20 Monate, seit 2006 im Auftrag des Senior Experten Service, SES, Bonn). In beispielhafter Weise gelang der Aufbau einer Ambulanz für neurologisch kranke Kinder und Jugendliche in Kathmandu mit Außenstellen in allen Distrikten. Zusätzlich folgten 2007-2012 mehrwöchige Tätigkeiten im Yemen, in Danyang, China, sowie in mehreren Waisenhäusern für behinderte Kinder und Jugendliche in $\mathrm{Ba}$ $\mathrm{ku}$, Aserbaidschan, und in der Mongolei.

Bei all diesen Auslandstätigkeiten vermittelte Gunter Gross-Selbeck gemeinsam mit seiner ebenso engagierten Frau Claudia Gross-Selbeck (Physiotherapieausbilderin) Wissen zu Diagnostik und Therapie neurologisch erkrankter Säug- linge, Klein- und Schulkinder (mit motorischen Störungen, mentalen Behinderungen sowie anderen Entwicklungsstörungen, Epilepsien, Störungen der Sinnesorgane, Muskelerkrankungen und mehr). Die Ausbildung von Ärzten, Schwestern und Therapeuten vor Ort war den beiden ebenso wichtig wie die Unterstützung beim Aufbau organisatorischer Strukturen mit dem Ziel nachhaltiger Selbstständigkeit der unterstützten Einrichtungen.

Der Vorstand der Deutschen Gesellschaft für Epileptologie e. V. (DGfE) freut sich sehr, Herrn Prof. Dr. Gross-Selbeck als Ehrenmitglied in seinen Reihen zu wissen.

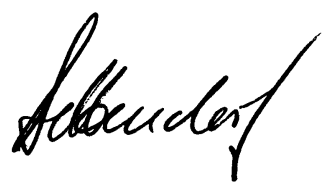

Dr. Bettendorf

\section{Korrespondenzadresse}

Dr. U. Bettendorf

Schwerpunktpraxis Epileptologie

Pestalozzistr. 4, 96114 Hirschaid

u.bettendorf@kiprahi.de

\section{Einhaltung der ethischen Richtlinien}

Interessenkonflikt. U. Bettendorf gibt an, dass kein Interessenkonflikt besteht. 